\title{
Cirugía de catarata traumática en pacientes pediátricos. Experiencia de un centro
}

\author{
Traumatic cataract surgery in pediatric patients. Experience in a site
}

\author{
Dra. Verónica Y. Burgos-Elías ${ }^{a}$ Dra. Ma. José Marroquín-Sarti ${ }^{a}$ Dr. Martin A. Zimmermann-Paiz ${ }^{b}$ \\ Dra. Ana Marissa Ordoñez Rivas ${ }^{b}$ Dra. Nancy C. Quezada-del Cidb
}

\section{RESUMEN}

El trauma ocular es un problema significativo en todo el mundo. El objetivo de este estudio fue determinar las características de los pacientes menores de 14 años operados de catarata traumática en la Unidad de Oftalmología Pediátrica, Estrabismo y Neurooftalmología "Dra. Ana María Illescas Putzeys", Hospital de Ojos y Oídos “Dr. Rodolfo Robles V.".

Se realizó un estudio retrospectivo, descriptivo, observacional, en el que se revisaron expedientes de pacientes operados de catarata de 2010 a 2015. Se operaron 54 niños por catarata traumática, de los cuales $75 \%$ eran hombres. El $68 \%$ pertenecían al rango de edad de 6-11 años. El tipo de trauma fue cerrado en un $57,4 \%$. Los pacientes que lograron una agudeza visual igual a $20 / 70$ o mayor fueron el $68,2 \%$.

Palabras clave: catarata, pediatría, traumatismo, cirugía, ambliopía.

http: / / dx.doi.org/10.5546/ aap.2018.216

Texto completo en inglés:

http: / / dx.doi.org/10.5546/ aap.2018.eng.216

Cómo citar: Burgos-Elías VY, Marroquín-Sarti MJ, Zimmermann-Paiz MA, et al. Cirugía de catarata traumática en pacientes pediátricos. Experiencia de un centro. Arch Argent Pediatr 2018;116(3):216-219.

a. Fellowship II en la Unidad de Oftalmología Pediátrica, Estrabismo y Neurooftalmología “Dra. Ana María Illescas Putzeys", Hospital de Ojos y Oídos "Dr. Rodolfo Robles V.", Instituto de Ciencias de la Visión, Benemérito Comité Pro Ciegos y Sordos de Guatemala, Guatemala.

b. Unidad de Oftalmología Pediátrica, Estrabismo y Neurooftalmología "Dra. Ana María Illescas Putzeys", Hospital de Ojos y Oídos "Dr. Rodolfo Robles V.", Instituto de Ciencias de la Visión, Benemérito Comité Pro Ciegos y Sordos de Guatemala, Guatemala.

Correspondencia:

Dra. Verónica Y. Burgos Elías: veronicaburgos1@hotmail.com

Financiamiento: Ninguno.

Conflicto de intereses: Ninguno que declarar.

Recibido: $17-5-2017$

Aceptado: 19-9-2017

\section{INTRODUCCIÓN}

El trauma ocular es un problema significativo en todo el mundo y es la principal causa de ceguera unilateral no congénita en niños. La incidencia de alteración visual grave o ceguera causada por trauma ocular en edad pediátrica varía de $2 \%$ a $14 \%$ según lo recopilado en diferentes estudios. ${ }^{1}$

Debido a lo anterior, globalmente, hay 3,9 millones de personas con baja visión bilateral o ceguera y más de 18 millones con alteración visual unilateral. ${ }^{2}$ La información en países en vías de desarrollo es escasa.

El desarrollo de catarata es una de las consecuencias serias postrauma ocular en adultos y niños; forma una categoría especial, ya que se presenta asociada a otras alteraciones oculares, tales como lesiones corneales, en el iris, hemorragia vítrea y desprendimiento de retina. ${ }^{3}$

El objetivo de este estudio fue determinar las características de los pacientes menores de 14 años operados de catarata traumática en la Unidad de Oftalmología Pediátrica, Estrabismo y Neurooftalmología “Dra. Ana María Illescas Putzeys", Hospital de Ojos y Oídos "Dr. Rodolfo Robles V."

\section{MATERIAL Y MÉTODOS}

Se realizó un estudio retrospectivo, descriptivo, observacional. Se revisaron los expedientes después de la autorización por parte de la Institución.

De los pacientes que fueron operados por catarata traumática de 2010 a 2015, se recabó la siguiente información: edad, género, tipo de lesión (trauma abierto o cerrado), intervalo de tiempo entre el trauma y la intervención, implante o no de lente intraocular (LIO), tratamiento o no de ambliopía, seguimiento posoperatorio y complicaciones. En los pacientes que cumplieron un año de seguimiento posoperatorio, también se documentó la agudeza visual (AV) pre- y posoperatoria (la cual fue medida con cartilla de Snellen en pacientes verbales y, en preverbales, la connotación de centra, sigue y mantiene [CSM]). 
La técnica quirúrgica utilizada fue la descrita en el Manual de Catarata en la Niñez, de Orbis International, ${ }^{4}$ que consiste en la creación del surco escleral, aspiración de catarata más capsulotomía posterior con vitrectomía anterior. Se implantó LIO en pacientes mayores de 2 años y con buen soporte capsular en el mismo tiempo quirúrgico.

Se tomó como un buen resultado visual una AV mejor o igual a 20/70 al año posoperatorio sobre la base de la mayoría de los estudios publicados respecto a catarata traumática en niños. ${ }^{5-7}$

Los pacientes operados que tuvieron una diferencia de 2 líneas de visión entre cada ojo recibieron tratamiento de ambliopía. Este consistió en parchar el ojo dominante por un mínimo de 8 horas al día con chequeos periódicos de 1 semana por cada año de edad con una duración mínima de tratamiento de 6 meses.

Los datos se consignaron y analizaron en una hoja diseñada para el efecto utilizando el programa Epi Info (Database and statistics software for public health professionals, Centers for Disease Control and Prevention 2008).

\section{RESULTADOS}

Durante el período del estudio, se les realizó cirugía de catarata a 207 pacientes. De estos, $54(26 \%)$ correspondían a catarata traumática.
En la Tabla 1, se hace un resumen de las características de los 54 pacientes; 41 de estos fueron del género masculino $(75 \%)$. Del total, $83 \%$ eran del área rural. El mayor porcentaje de pacientes estuvo en el rango de edad de 6 a 11 años (68\%).

El principal tipo de trauma fue el cerrado en 31 pacientes $(57,4 \%)$ y se desconoció el mecanismo del trauma en su mayor porcentaje en 16 pacientes (29\%). En segundo lugar, se debió a una rama de árbol en 11 pacientes (20\%). Otros objetos: los punzocortantes (alambres, agujas, lapiceros, tijeras) en un $16,7 \%$, piedras en un $11,1 \%$ y otros (cincho, golpes, balón, limón, semilla de café) en un $23,2 \%$.

El intervalo de tiempo entre el trauma y la cirugía de catarata fue mayor de 30 días en el 87\% de los casos.

En este estudio, no hubo relación estadísticamente significativa $(\mathrm{p}=0,44)$ en cuanto al pobre resultado visual y el trauma abierto (42,9\%). Tampoco hubo relación estadísticamente significativa $(p=0,44)$ al comparar un buen resultado visual con trauma cerrado $(53,3 \%)$.

Solo 22 pacientes $(40,7 \%)$ cumplieron el seguimiento durante 1 año después de la cirugía.

En la Tabla 2, se resume la AV previa a la cirugía de catarata de estos 22 pacientes según el rango de edad.

TABLA 1.Total de pacientes con catarata traumática durante 2010-2015

\begin{tabular}{|c|c|c|c|c|c|c|c|c|c|c|c|}
\hline \multirow{2}{*}{$\begin{array}{l}\text { Edad } \\
\text { (años) }\end{array}$} & \multicolumn{2}{|c|}{ Género } & \multirow[t]{2}{*}{ Total $(\%)$} & \multirow[b]{2}{*}{ Cerrado } & \multirow[b]{2}{*}{ Abierto } & \multicolumn{4}{|c|}{ Intervalo entre accidente y cirugía (días) } & \multirow[b]{2}{*}{ Rural } & \multirow[b]{2}{*}{ Urbano } \\
\hline & $\mathbf{M}$ & $\mathbf{F}$ & & & & $0-2$ & $3-7$ & $8-30$ & $>30$ & & \\
\hline $0-2$ & 1 & 0 & $1(1,9)$ & 0 & 1 & 0 & 0 & 0 & 1 & 1 & 0 \\
\hline $3-5$ & 8 & 3 & $11(20,4)$ & 5 & 6 & 0 & 0 & 2 & 9 & 10 & 1 \\
\hline $6-8$ & 12 & 7 & $19(35,2)$ & 10 & 9 & 0 & 1 & 1 & 17 & 17 & 2 \\
\hline $9-11$ & 15 & 3 & $18(33,3)$ & 13 & 5 & 0 & 0 & 0 & 18 & 13 & 5 \\
\hline $12-14$ & 5 & 0 & $5(9,4)$ & 3 & 2 & 1 & 1 & 1 & 2 & 4 & 1 \\
\hline Total (\%) & $41(75,9)$ & $13(24,1)$ & $54(100)$ & $31(57,4)$ & $23(42,6)$ & $1(1,9)$ & $2(3,7)$ & $4(7,4)$ & $47(87,0)$ & $45(83,3)$ & $9(16,7)$ \\
\hline
\end{tabular}

M: masculino; F: femenino.

TABLA 2. Agudeza visual preoperatoria en pacientes con seguimiento de 1 año

\begin{tabular}{lccccccc}
\hline & \multicolumn{7}{c}{ Agudeza visual } \\
\cline { 2 - 6 } Edad (años) & $\mathbf{2 0 / 2 0 - 2 0 / 4 0}$ & $\mathbf{2 0 / 5 0 - 2 0 / 7 0}$ & $\mathbf{2 0 / 1 0 0 - 2 0 / 2 0 0}$ & $\mathbf{2 0 / 4 0 0 - M M}$ & PL & NPL & Total (\%) \\
\cline { 2 - 7 } & 0 & 0 & 0 & 0 & 1 & 0 & $1(4,4)$ \\
$0-2$ & 0 & 0 & 0 & 3 & 3 & 0 & $6(27,7)$ \\
$3-5$ & 0 & 0 & 2 & 2 & 2 & 0 & $6(27,3)$ \\
$6-8$ & 0 & 0 & 1 & 4 & 0 & 0 & $5(22,7)$ \\
$9-11$ & 0 & 0 & 1 & 0 & 3 & 0 & $4(18,2)$ \\
$12-14$ & 0 & 0 & $4(18,2)$ & $9(40,9)$ & $9(40,9)$ & 0 & $22(100)$ \\
Total $(\%)$ & 0 & & & & &
\end{tabular}

MM: movimiento de manos; PL: percepción de luz; NPL: no percepción de luz. 
En la Tabla 3, se observa la AV final (al año de seguimiento) dividida en rango de edad.

El intervalo de tiempo entre el trauma y la cirugía de catarata de los pacientes que llevaron seguimiento fue mayor de 30 días en 17 casos $(77,3 \%)$.

En esta serie, se implantó un LIO en un $77,3 \%$ de los pacientes. En 14 de estos niños $(63,6 \%)$, se obtuvo buen resultado visual, que fue estadísticamente significativo $(\mathrm{p}=0,01)$. En cuanto a los 5 pacientes áfacos, solamente uno tuvo buen resultado visual (AV 20/25), relacionado con el uso constante de sus gafas.

De los 22 casos, solo $7(31,8 \%)$ requirieron tratamiento de ambliopía.

Únicamente, 6 pacientes $(27,3 \%)$ de los que cumplieron el seguimiento presentaron complicaciones: glaucoma (en un paciente áfaco y uno pseudofaco), un caso presentó ruptura de una háptica del LIO y quedó áfaco, uno con desprendimiento de retina, un caso de opacidad corneal grave y un paciente con endoftalmitis (presente antes de la cirugía), que fue el único con AV final de no percepción de luz.

\section{DISCUSIÓN}

El resultado visual en pacientes pediátricos con catarata traumática depende de múltiples factores, tales como edad en la que ocurre el insulto visual, tipo de lesión ocular y tratamiento (procedimiento quirúrgico, corrección del defecto refractivo, tratamiento de ambliopía y apego al tratamiento).

En esta revisión, la mayoría de los pacientes fueron de género masculino $(75,9 \%)$, lo que coincidió con la mayoría de estudios realizados en Latinoamérica, Europa y Asia, donde este porcentaje osciló entre el $70 \%$ y el $85 \% .^{5-7}$ Esto podría deberse a que los niños realizaban actividades de mayor peligro y fuerza.,
El grupo de edad que se relacionó con más frecuencia fue de 6-11 años, igual que en otros países que reportaron edad promedio de 6,610 años..$^{5-7}$

El tipo de trauma más frecuente en nuestra serie fue el cerrado en $57,4 \%$ y el abierto en $42,6 \%$; esto coincidió con lo reportado en otros estudios, en los que el trauma cerrado osciló entre un $48 \%$ y un $87 \%$, y el trauma abierto, entre un $19,6 \%$ y un $39 \% .3,9,10$

Entre los objetos causales del trauma, se encontró la rama de árbol en un $20 \%$, que fue similar a un estudio en el que se reportó en un $28 \%$, y los objetos punzocortantes (alambres, agujas, lapiceros, tijeras) en un $39 \%$, que fue mucho mayor del 16,7\% encontrado en esta serie. ${ }^{3}$

En la mayoría de los estudios, se toma como base un buen resultado visual mejor o igual a 20/70.-7 Una AV mejor o igual a 20/40 o mejor se ha reportado en un $45-70 \%$ de los niños después de la extracción de catarata traumática. ${ }^{3,6,7,11}$ En esta serie, 15 pacientes que cumplieron un año de seguimiento lograron una AV mejor que 20/70. De ellos, 12 pacientes tuvieron una AV mejor que $20 / 40$ y 3 pacientes, en el rango de $20 / 50$ a 20/70.

Es probable que el buen resultado visual se debiera a que las secuelas del trauma no ocasionaron cambios estructurales de importancia tanto a nivel de segmento anterior (córnea) como segmento posterior (retina).

$\mathrm{Al}$ igual que en otras publicaciones, en nuestro estudio, la mayoría de los niños consultó en un tiempo mayor de 30 días luego del trauma. ${ }^{6,7,12}$

El tratamiento quirúrgico con implante de LIO para niños con catarata traumática es efectivo para la rehabilitación visual. ${ }^{11,13}$ En Israel, se encontró significancia estadística en cuanto al pobre resultado visual en ojos áfacos comparados con pseudofacos. ${ }^{11}$

TABla 3. Agudeza visual posoperatoria en pacientes con seguimiento de 1 año

\begin{tabular}{|c|c|c|c|c|c|c|c|}
\hline \multirow[b]{3}{*}{ Edad (años) } & \multicolumn{6}{|c|}{ Agudeza visual } & \multirow[b]{3}{*}{ NPL } \\
\hline & \multicolumn{6}{|c|}{ Posoperatoria (N: 22) } & \\
\hline & $20 / 20-20 / 25$ & $20 / 30-20 / 40$ & 20/50-20/70 & $20 / 100-20 / 200$ & 20/400-MM & PL & \\
\hline $0-2$ & 0 & 1 & 0 & 0 & 0 & 0 & 0 \\
\hline 3-5 & 0 & 5 & 1 & 0 & 0 & 0 & 0 \\
\hline 6-8 & 1 & 2 & 0 & 2 & 1 & 0 & 0 \\
\hline $9-11$ & 2 & 0 & 1 & 0 & 1 & 1 & 0 \\
\hline $12-14$ & 0 & 1 & 1 & 1 & 0 & 0 & 1 \\
\hline TOTAL (\%) & $3(13,6)$ & $9(40,9)$ & $3(13,6)$ & $3(13,6)$ & $2(9,1)$ & $1(4,6)$ & $1(4,6)$ \\
\hline
\end{tabular}

MM: movimiento de manos; PL: percepción de luz; NPL: no percepción de luz. 
Treinta y dos pacientes de 54 no completaron un año de seguimiento, lo cual podría deberse a que, en su mayoría, provenían del área rural del país (lejanía de la ciudad capital, difícil acceso a las comunidades, pobreza extrema). Esto fue un factor de mal pronóstico tomando en cuenta la necesidad de tratamiento de ambliopía y corrección óptica en algunos de estos pacientes.

En uno de los estudios revisados, recomiendan las siguientes medidas para el manejo de trauma ocular: $^{8}$

a) Determinar rápidamente el alcance de la lesión para evitar ocasionar más daño.

b) Si se sospecha que el globo o el párpado están lacerados, colocar una concha plástica (o un vaso de papel sujeto con cinta), lo cual evitará el frote.

c) Referencia inmediata a un centro especializado.

\section{CONCLUSIÓN}

La edad de mayor incidencia del trauma fue de 6 a 11 años; el género masculino fue el más afectado y, de estos, la mayoría tuvo trauma cerrado. En esta serie, 15 de los pacientes con catarata traumática que cumplieron 1 año de seguimiento lograron una AV mejor o igual que $20 / 70$.

\section{REFERENCIAS}

1. Al-Mahdi HS, Bener A, Hashim SP. Clinical pattern of pediatric ocular trauma in fast developing country. Int Emerg Nurs 2011;19(4):186-91.

2. AbbottJ,Shah P. The epidemiology and etiology of pediatric ocular trauma. Surv Ophthalmol 2013;58(5):476-85.
3. Gogate $\mathrm{P}$, Sahasrabudhe $\mathrm{M}$, Shah $\mathrm{M}$, et al. Causes, epidemiology, and long-term outcome of traumatic catarcts in children in rural India. Indian J Ophthalmol 2012;60(5):4816.

4. Neely D, Wilson E, Plager D, et al. Cataracts in Childhood. Orbis International. 2016. [Acceso: 7 de marzo 2017]. Disponible en: https: / d1dk84hmsdb5u9.cloudfront. net /991974c8-8e3f-431f-82ec-21b0fb7c92dc/ Resources / Cataracts $\% 20$ in $\% 20$ Children $\% 20$ Manual.pdf.

5. Shah M, Shah S, Applewar A, et al. Ocular Trauma Score as a predictor of final visual outcomes in traumatic cataract cases in pediatric patients. J Cataract Refract Surg 2012; 38(6):959-65.

6. Reddy A, Ray R, Yen K. Surgical intervention for traumatic cataracts in children: Epidemiology, complications, and outcomes. J AAPOS 2009;13(2):170-4.

7. Xu Y, Huang $\mathrm{Y}, \mathrm{Xie}$ L. Pediatric traumatic cataract and surgery outcomes in eastern China: a hospital-based study. Int J Ophthalmol 2013;6(2):160-4.

8. Quezada-del Cid N, Zimmermann-Paiz M, OrdoñezRivas A. Características clínicas y epidemiológicas del trauma ocular en menores de 14 años. Arch Argent Pediatr 2015;113(5):e260-63.

9. Pons-Castro L, Arias-Díaz A, Naranjo Fernández R, et al. Resultados del tratamiento quirúrgico de la catarata traumática en edad pediátrica. Rev Cuba Oftalmol 2010;23(2)209-218.

10. Razo-Blanco-Hernández DM, Pérez-Bastida XI, SánchezNava MF, et al. Estimación del daño por trauma ocular en pediatría sin la evaluación de la agudeza visual. Bol Med Hosp Infant Mex 2011;68(5):337-41.

11. Rumelt S, Rehany U. The influence of surgery and intraocular lens implantation timing on visual outcome in traumatic cataract. Graefes Arch Clin Exp Ophthalmol 2010;248(9):1293-7.

12. Shah M, Shah S, Upadhyay P, Agrawal R. Controversies in traumatic cataract classification and management: a review. Can J Ophthalmol 2013;48(4):251-8.

13. Shah M, Shah S, Shikhangi K, et al. Factors Affecting Visual Outcome Following Surgical Treatment of Cataracts in Children. Open J Ophthalmol 2012;2(4):131-9.

\title{
Prevalencia de alergia a la proteína de la leche de vaca en niños en un hospital universitario de comunidad Prevalence of cow's milk protein allergy among children in a university community hospital
}

\author{
Dra. Romina Mehaudy, ${ }^{a, b}$ Dr. Claudio A. S. Parisi, ${ }^{c, b, f}$ Dra. Natalia Petriz, ${ }^{c, b}$ Dr. Alfredo Eymann, ${ }^{d}$ \\ Lic. María B. Jaureguie, y Dra. Marina Orsia,
}

a. Servicio de Gastroenterología Infantil.

b. Consultorio Interdisciplinario de Alergias Alimentarias.

c. Sección Alergia Pediátrica, Servicio de Clínica Pediátrica.

d. Servicio de Clínica Pediátrica.

e. Servicio de Alimentación

Hospital Italiano de Buenos Aires.

f. Grupo de Trabajo Alérgeno Alimentario, Instituto Internacional de Ciencias de la Vida (ILSI). Argentina.
Correspondencia:

Dr. Claudio A.S. Parisi: claudio.parisi@hospitalitaliano.org.ar

Financiamiento: Ninguno.

Conflicto de intereses: Ninguno que declarar.

Recibido: 14-8-2017

Aceptado: 26-10-2017 